> Les télomères sont des structures nucléoprotéiques finement régulées qui constituent les extrémités des chromosomes linéaires. Chez I'homme, les défauts de maintenance télomérique sont responsables de la dyskératose congénitale (DC), une maladie génétique rare caractérisée par une aplasie médullaire, un vieillissement prématuré et une prédisposition aux cancers. Des données récentes qui proviennent d'une étude de patients atteints du syndrome de HoyeraalHreidarsson (syndrome $H H$ ), variant sévère de $D C$, ont mis en évidence la grande hétérogénéité moléculaire de cette maladie, dont certaines formes sont liées à des défauts de facteurs impliqués, non pas dans la régulation de la taille des télomères, mais dans leur protection et leur réplication. <

Les télomères : des structures ribonucléoprotéiques complexes finement régulées

Les télomères constituent les extrémités des chromosomes linéaires et sont composés, chez les vertébrés, de répétitions de séquences hexamériques TTAGGG. Ils forment une structure unique qui interagit avec des facteurs dont le rôle est de protéger l'extrémité chromosomique de la dégradation et/ou de la fusion. Au cours des divisions cellulaires, les ADN polymérases conventionnelles sont incapables de répliquer totalement la fin des chromosomes, ce qui conduit à une perte progressive des séquences télomériques. Si les télomères atteignent une taille trop courte, les cellules stoppent leur prolifération et entrent dans un état de sénescence réplicative et/ou en apoptose [1]. Ce processus d'arrêt réplicatif, qui dépend de la protéine proapoptotique $\mathrm{p} 53$, prévient l'instabilité chromosomique et les proliférations cellulaires incontrôlées. Pour contrer le raccourcissement des télomères, la télomérase TERT (telomerase reverse transcriptase), une transcriptase inverse, ajoute des répétitions TTAGGG au cours de chaque division cellulaire. L'expression

\section{Nouvelles formes de dyskératoses congénitales}

Le dysfonctionnement des télomères n'est pas systématiquement associé à une réduction de leur taille

Fabien Touzot, Tangui Le Guen, Jean-Pierre de Villartay, Patrick Revy

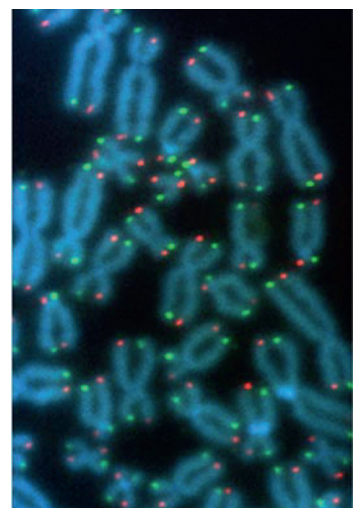

Inserm U768, équipe dynamique du génome et système immunitaire (DGSI) ; hôpital Necker-Enfants malades, 149, rue de Sèvres 75015, Paris, France; fondation Imagine, Paris, France. patrick.revy@inserm.fr fabien.touzot@inserm.fr

de TERT est finement régulée et principalement limitée aux cellules germinales, aux cellules souches hématopoiétiques ainsi qu'à certaines cellules activées (comme les lymphocytes) [2]. La plupart des cellules somatiques, comme les fibroblastes, n'expriment pas ou peu la molécule TERT et, par conséquent, ont une capacité proliférative limitée [3]. La télomérase agit sous forme d'un complexe moléculaire composé de nombreux autres facteurs dont la dyskérine, la matrice d'ARN TERC (telomerase RNA component) et les cofacteurs NOP10 (nucleolar protein 10), NHP2 (non-histone chromosome protein 2), TCABI (telomerase Cajal body protein 1) et GARl. Le rôle du raccourcissement télomérique dans l'induction de la sénescence et la limitation de la prolifération cellulaire a été clairement démontré. En effet, l'immortalisation de fibroblastes primaires peut être induite par l'expression ectopique de TERT, le composant transcriptase inverse de la télomérase, permettant le maintien d'une taille stable des télomères au cours des divisions successives [4]. De plus, la plupart des cellules tumorales réexpriment TERT (dans plus de $90 \%$ des cancers humains), leur conférant un potentiel de division infini propre au phénomène d'immortalisation [5].

La taille et la stabilité des télomères sont également contrôlées par des facteurs télomériques spécifiques - TRF1, TRF2, RAP1 (Repressor/ activator protein 1 homolog), POTl (protection of telomere 1 protein), TPP1 (TIN2 and POT1 organizing protein) et TIN2 (TRF1-interacting protein 2) - formant le complexe «shelterine» [6]. Ces facteurs 


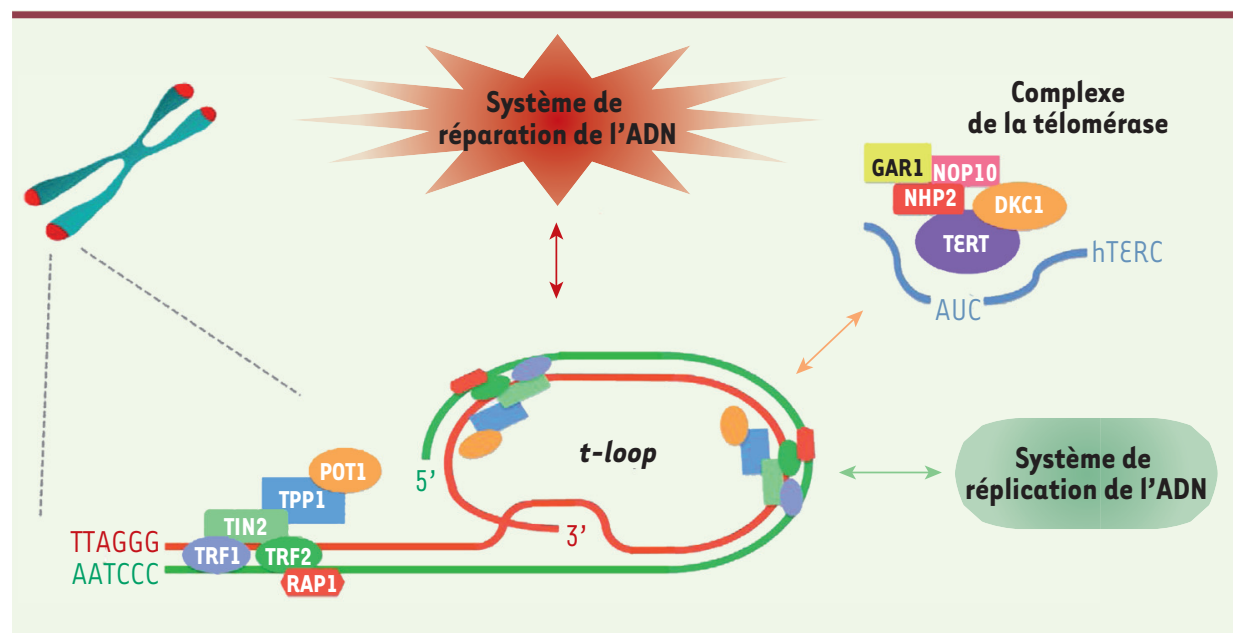

Figure 1. Structure des télomères. Les télomères sont les extrémités des chromosomes linéaires. Ils sont constitués de répétitions d'ADN hexamérique (TTAGGG)n s'étendant chez l'homme sur 5 à 15 kilobases, et se terminant en 3' par un surplomb simple brin de 50 à 500 nucléotides capable d'envahir l'ADN télomérique double brin pour former une structure appelée t-loop. L'ADN télomérique recrute six facteurs spécifiques dédiés à la protection des extrémités télomériques, les shelterines : TRF1, TRF2, TIN2, POT1, RAP1, TPP1. Ces facteurs contribuent à l'organisation conformationnelle des télomères et régulent le recrutement des différents systèmes interagissant avec les télomères : le complexe télomérase et les systèmes de réplication et de réparation de l'ADN.

permettent de structurer les télomères, contrôlent le recrutement du complexe télomérase et empêchent les voies de réparation de l'ADN de reconnaître les extrémités des chromosomes comme des cassures double-brin de l'ADN (Figure 1).

Associés aux shelterines, de nombreux facteurs de réparation de l'ADN, dont Ku70/Ku80, ATM (ataxia telangiectasia mutated), ATR (ataxia telangiectasia mutated and Rad3 related), DNA-PKc (DNA-dependent protein kinase, catalytic subunit), Mrell (meiotic recombination 11 homolog A)/Rad50/NBS1 (Nijmegen breakage syndrome 1) (ou $M R N$ complex) et Apollo participent également à la maintenance télomérique [1].

\section{La dyskératose congénitale : modèle humain de dysfonctionnement télomérique}

Un dysfonctionnement télomérique, causé soit par une diminution trop importante de la taille des télomères, soit par un défaut de la cape de protection, provoque l'activation des voies de réparation de l'ADN, qui reconnaissent alors les extrémités chromosomiques comme des cassures double-brin de l'ADN. Ceci conduit à l'activation de nombreuses molécules impliquées dans la signalisation et la réparation des dommages de I'ADN (ATM, ATR, le complexe MRN, 53BPl [p53 binding protein 1], etc.) [1]. L'activation des voies de réparation de l'ADN est responsable de l'entrée de la cellule en sénescence, de son apoptose et des fusions entre les chromosomes, fusions qui génèrent des chromosomes dicentriques et sont donc responsables d'instabilité génétique.

Le rôle essentiel de la maintenance télomérique est clairement mis en évidence dans des maladies génétiques rares associées à des défauts de protection et/ou de taille des télomères : les dyskératoses congé- nitales (DC). Depuis leur première description en 1910 par $\mathrm{F}$. Zinsser ${ }^{1}$, le diagnostic de DC a longtemps reposé sur la triade pathognomonique : pigmentation anormale de la peau, dystrophie unguéale et leucoplasie orale. Par la suite, la sémiologie de la maladie s'est grandement étoffée par la description d'atteintes organiques diverses: aplasie médullaire, fibrose pulmonaire, cirrhose hépatique, entéropathie avec malabsorption, sténose œsophagienne, atrophie cérébelleuse [7, 8].

Les signes cliniques des formes classiques de DC se manifestent le plus souvent dans l'enfance. Les anomalies phanériennes apparaissent généralement en premier, avant l'âge de 10 ans. L'insuffisance médullaire, complication majeure de la maladie, se développe souvent avant l'âge de 20 ans, $90 \%$ des patients souffrant d'aplasie médullaire à l'âge de 30 ans. Cependant, il existe des variations considérables dans l'âge d'apparition et la gravité de la maladie d'un patient à l'autre, parfois au sein d'une même famille. Les principales causes de mortalité chez les patients atteints de DC sont l'insuffisance médullaire, le déficit immunitaire (60 à $70 \%$ ), les complications pulmonaires (10 à $15 \%$ ) et les tumeurs malignes (10\%) $[7,8]$.

Le syndrome de Hoyeraal-Hreidarsson (syndrome $\mathrm{HH}$ ) est une forme rare et sévère de DC, associant au moins quatre des six caractéristiques suivantes: une microcéphalie, une hypoplasie cérébelleuse, un retard de croissance à début anténatal, un retard de développement, un déficit immunitaire et une aplasie médullaire [9]. Signe de la sévérité de ce syndrome, l'insuffisance médullaire qui apparaît dans les premières années de vie est responsable du décès des patients avant l'âge de dix ans.

\section{Caractérisation génétique de la dyskératose congénitale et du syndrome de Hoyeraal-Hreidarsson}

Les causes moléculaires de $D C$ sont très hétérogènes, tant sur le plan des gènes incriminés, du type de

\footnotetext{
${ }^{1}$ Pour plus d'informations, voir [35].
} 
mutation retrouvée, que du mode de transmission - transmission liée à I'X, autosomique dominante ou autosomique récessive -. Les corrélations génotype/phénotype sont loin d'être parfaites et la gravité de la maladie chez certains patients reste mal comprise.

Par ailleurs, si actuellement de nombreux gènes impliqués dans la survenue des DC ont été identifiés, $60 \%$ des patients présentant les caractéristiques cliniques de $\mathrm{DC}$ ou de syndrome $\mathrm{HH}$ restent encore sans diagnostic moléculaire.

\section{Formes liées au chromosome $X$}

L'identification par clonage positionnel du gène responsable de la forme de la maladie récessive liée au chromosome $X$ - le gène $D K C 1$ codant la protéine dyskérine $[10,11]$ - a permis d'établir un lien entre $D C$ et défaut de maintenance télomérique. En effet, la dyskérine est une composante essentielle du complexe de la télomérase, et la plupart des mutations identifiées sont localisées dans le domaine de liaison à la matrice ARN hTERC [12]. Une déficience en dyskérine pourrait entraîner une diminution de la stabilité de la matrice ARN hTERC [13].

\section{Formes autosomiques dominantes}

Le lien entre $D C$ et dysfonctionnement télomérique s'est trouvé renforcé par l'identification de mutations dans d'autres composantes du complexe télomérase dont la matrice ARN hTERC [14]. Des délétions de hTERC sont responsables des formes autosomiques dominantes de DC. Des mutations ponctuelles peuvent également modifier la structure tertiaire de l'ARN affectant l'activité ou la « processivité ${ }^{2}$ » de la télomérase, ou plus simplement la stabilité de hTERC [15].

Des mutations de la sous-unité catalytique du complexe télomérase hTERT provoquent également une DC de transmission autosomique dominante. Elles se situent tout au long de la séquence codante et sont susceptibles d'influer sur les trois domaines de hTERT : la région amino-terminale, les motifs de la transcriptase inverse ou le domaine carboxy-terminal. L'étude de 41 personnes présentant une délétion dans le bras court du chromosome 5 entraînant une haplo-insuffisance pour hTERT a permis de montrer que l'attrition télomérique chez les patients haplo-insuffisants, bien que légèrement accélérée, n'était pas suffisante pour provoquer un phénotype de DC en l'absence de phénomène d'anticipation (voir plus loin ) [16].

Des mutations hétérozygotes du gène TINF2 codant la shelterine TIN2, pour la plupart apparues de novo, sont responsables d'environ $10 \%$ des cas de DC. Chez les patients déficients en TIN2, la maladie s'exprime de manière particulièrement sévère et se développe précocément [17]. Il est intéressant de noter que les mutations de TIN2 sont très majoritairement localisées dans une région comprise entre les acides aminés 270 à 300 , à proximité du domaine PXVXL nécessaire à l'interaction de TIN2 avec la protéine de l'hétérochromatine HPl $\gamma$. Ces mutations abrogent la fixation de HPl $\gamma$ à TIN2, entraînant un défaut de cohésion des télomères sœurs [18]. Elles provoquent également une diminution de la capacité de TIN2 à interagir avec le complexe

${ }^{2}$ Terme employé pour les enzymes qui présentent une activité qui peut être répétée. télomérase, pouvant ainsi compromettre le recrutement de ce dernier aux télomères [19].

\section{Formes récessives autosomiques}

Des mutations de NOP10 [20] et NHP2 [21] sont responsables de $D C$ par transmission autosomique récessive. Dyskérine, NOP10 et NHP2 sont associés à la fois au complexe de la télomérase et aux ribonucléoprotéines snoRNP (small nucleolar ribonucleoproteins) H/ACA qui sont des complexes nucléolaires catalysant spécifiquement la pseudo-uridylation des ARN ribosomaux. Plus récemment, des mutations hétérozygotes composites faux-sens de TCABl, facteur impliqué dans la localisation de la télomérase aux corps de Cajal, ont été mises en évidence chez deux patients atteints de DC. Ces mutations entraînent un défaut de la localisation de la télomérase aux corps de Cajal et une accumulation de hTERC dans les nucléoles, conduisant à un défaut d'élongation des télomères [22].

Tous les gènes reponsables de DC décrits ci-dessus participent donc, directement ou indirectement, à l'activité du complexe télomérase aux télomères. Par conséquent, les $D C$ causées par des mutations de ces gènes sont invariablement associées à des raccourcissements accélérés des télomères.

Dans un sous-groupe de DC de transmission autosomique récessive (dont le phénotype semble peu sévère), des mutations bialléliques du gène $\mathrm{Cl6orf57}$ ont été mises en évidence [23]. Cependant, aucun signe de dysfonctionnement télomérique n'a été observé chez ces patients dont les télomères sont de taille normale. La fonction du gène C16orf57 reste encore inconnue.

\section{Longueur des télomères et diagnostic de $D C$}

La mesure de la longueur des télomères dans les cellules du sang périphérique constitue à ce jour le test de dépistage de référence pour l'identification des patients atteints de DC du fait de la relation communément admise entre DC/aplasie médullaire et télomères anormalement courts [24]. Cette mesure peut s'effectuer par Southern blot, PCR (polymerase chain reaction) quantitative ou FISH (fluorescence in situ hybridization) quantitatif parfois couplé à la cytométrie de flux (flow-FISH) [24-26]. La longueur des télomères des leucocytes diminuant avec l'âge chez les individus sains, ce test diagnostic se révèle plus discriminant chez les enfants que chez les adultes [26].

Chez les patients atteints de DC sans aplasie médullaire, les télomères sont plus courts que la moyenne, mais leur longueur est parfois comprise dans la distribution des 


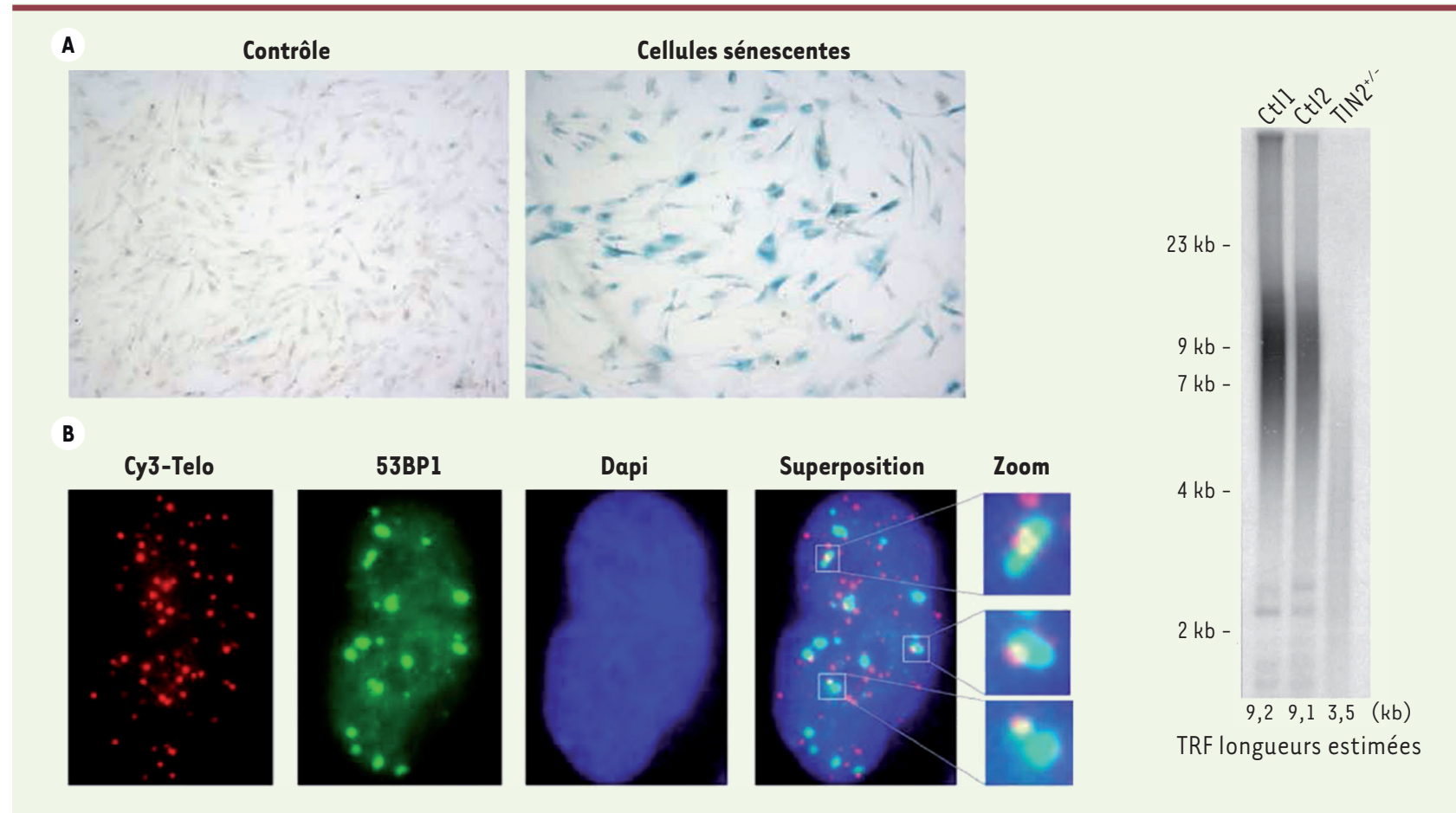

Figure 2. Tests utilisés pour détecter les dysfonctionnements télomériques dans les fibroblastes primaires de patients atteints de syndrome HH. A. La sénescence réplicative précoce, conséquence du dysfonctionnement télomérique, est mise en évidence par la détection de l'activité $\beta$-galactosidase (SA- $\beta$-Gal) à pH acide. B. Les foyers de dysfonctionnement télomérique (TIF pour telomere dysfunction-induced foci) correspondent à la colocalisation, en immunofluorescence et FISH, du facteur de réparation 53BPl et des télomères révélés par une sonde fluorescente spécifique (Cy3-Telo). C. La mesure des télomères est réalisée par southern blot à l'aide d'une sonde spécifique des télomères après digestion de l'ADN génomique. Sont représentés ici les résultats obtenus avec de l'ADN issu de deux individus sains (CtIl et CtI2) et un patient HH (TIN2 ${ }^{+/-}$) ayant une mutation de TIN2 et des télomères anormalement courts.

tailles télomériques de la population générale. Ce test est donc peu sensible pour distinguer un patient d'un individu sain. Ceci est particulièrement vrai au sein des familles de patients atteints de $D C$ chez qui les télomères courts, mais non la mutation pathogène, peuvent être hérités du parent atteint [24]. Ainsi, en l'absence d'aplasie médullaire, les tests génétiques restent incontournables pour affirmer le diagnostic de DC.

Les formes de $D C$ liées à des mutations des composants de la télomérase sont des maladies à anticipation, la sévérité de la pathologie s'accroissant génération après génération ${ }^{3}$ [27], suggérant un rôle critique de la taille des télomères dans l'expression phénotypique de la maladie. Cependant, il n'est actuellement toujours pas certain que la sévérité clinique de la maladie soit réellement corrélée à la taille des télomères, les données récentes publiées à ce sujet restant très contradictoires $[28,29]$.

\footnotetext{
${ }^{3}$ Lorsque les $D C$ sont causées par une mutation dominante dans un facteur régulant la taille des télomères, la première personne atteinte peut avoir une pathologie peu prononcée ( $D C$ ou fibrose pulmonaire) avec des télomères un peu plus courts que la moyenne. En revanche ses enfants héritent des télomères courts de ce parent atteint et peuvent également hériter de la mutation. Par conséquent la maladie devient de plus en plus sévère au fil des générations avec des télomères de plus en plus courts. Cependant, certaines $D C$ ne présentent pas ce phenomène d'anticipation (soit parce que les mutations ne sont pas dominantes soit parce qu'elles ne touchent pas un composant intervenant dans la régulation de la taille des télomères).
}

\section{Identification de nouvelles formes du syndrome de Hoyeraal-Hreidarsson associant dysfonctionnement des télomères dysfonctionnels et taille normale des télomères}

Nous avons récemment analysé le phénotype de fibroblastes de cinq patients répondant aux critères cliniques du syndrome $\mathrm{HH}$ afin de détecter d'éventuelles anomalies de protection et/ou de taille des télomères [30]. Les principaux tests réalisés sont la mesure du taux de sénescence (détectable par l'analyse de l'activité $\beta$-galactosidase en $\mathrm{pH}$ acide [Figure 2A]), la détection par immunofluorescence des foyers de dysfonctionnements télomériques (TIF pour telomere dysfunction-induced foci) matérialisés par la localisation aux télomères du facteur de réparation de I'ADN 53BPl (Figure 2B), ainsi que la mesure de la taille des télomères (mesurée par Southern blot après digestion de l'ADN extrait des fibroblastes et des cellules sanguines et hybridation avec une sonde spécifique des télomères [Figure $2 C]$ ). La présence d'aberrations télomériques peut être ensuite détectée par 


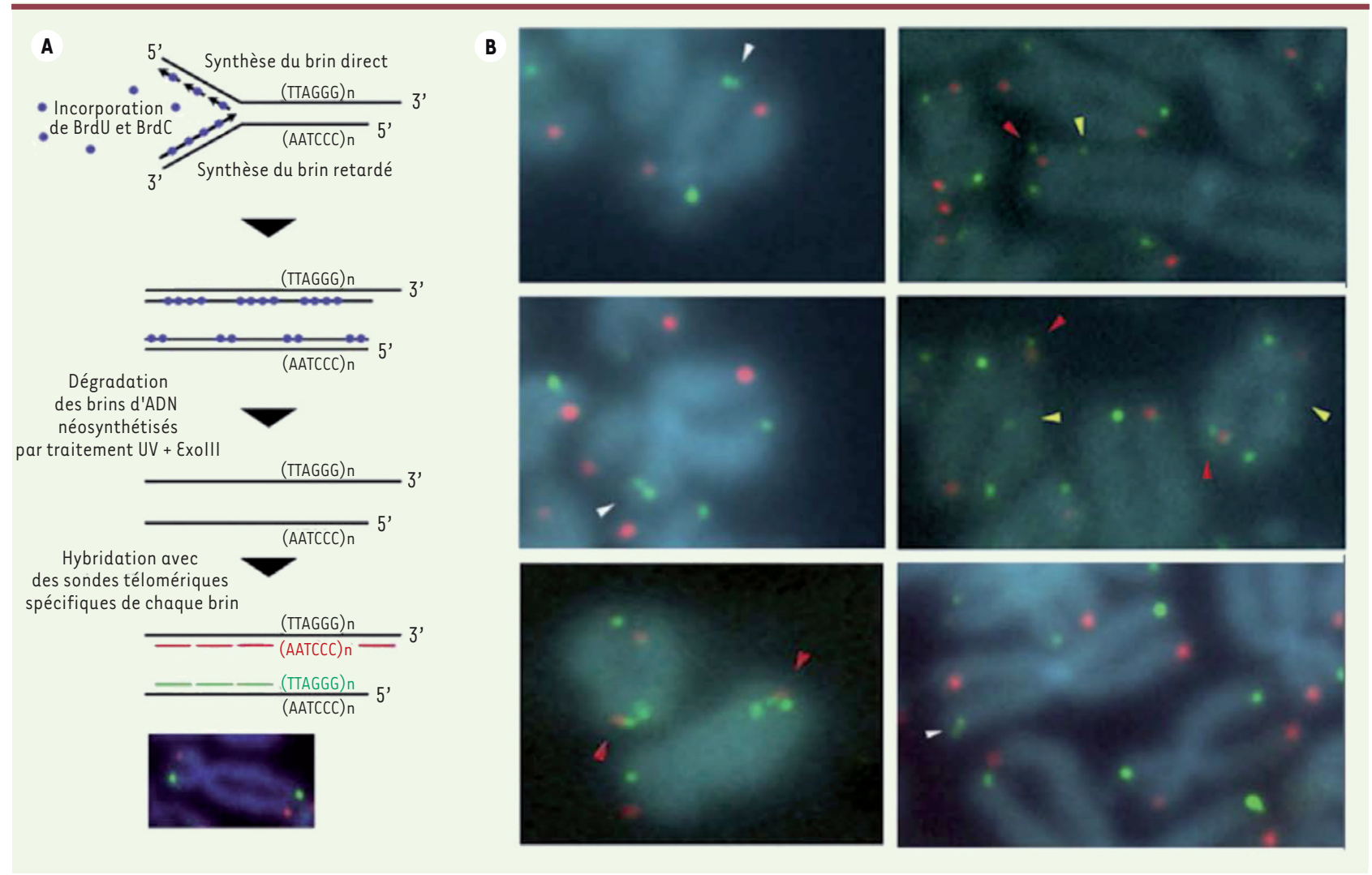

Figure 3. Aberrations télomériques dans les cellules de patients atteints de syndrome HH révélées par la technique de CO-FISH. A. Le CO-FISH est une technique de FISH réalisée après exposition des cellules à la bromodéoxyuridine (BrdU) et la bromodéoxycytidine (BrdC) pendant un cycle cellulaire. Un traitement par irradiation UV et digestion enzymatique (ExolII) permet la dégradation des brins nouvellement synthétisés. L’hybridation avec des sondes télomériques spécifiques des brins meneur (sonde verte) et retard (sonde rouge) permet de détecter les télomères issus de la synthèse directe et retardée. $B$. Le CO-FISH réalisé sur des fibroblastes de certains patients atteints de syndrome HH met en évidence des anomalies morphologiques télomériques franches: augmentation des doublets télomèriques avec préférence de brin (têtes de flèches blanches), présence de signaux télomériques interstitiels (têtes de flèches jaunes), présence de signaux télomériques multiples (têtes de flèches rouges). Ces anomalies suggèrent une fragilité des télomères lors du processus réplicatif.

des techniques de FISH et de FISH avec orientation chromosomique (CO-FISH) (Figure 3A).

L'analyse phénotypique des fibroblastes d'un des patients $\mathrm{HH}$, dont les caractéristiques cliniques avaient été précédemment publiées [31], a révélé des marques de déprotection télomérique (TIF, sénescence) sans que la taille des télomères ne soit affectée. Une analyse génétique de candidats impliqués dans la protection des télomères a permis d'identifier une anomalie d'épissage du préARNm d'Apollo, un facteur de réparation de l'ADN [32, 33]. Cette aberration d'épissage est à l'origine de l'expression d'une forme tronquée d'Apollo, qui perd un motif TBM (TRF binding motif) nécessaire à son interaction avec la shelterine TRF2 et, par conséquent, à sa localisation aux télomères. Cette forme tronquée d'Apollo garde cependant une capacité d'interaction avec la forme sauvage d'Apollo. Elle perturbe le recrutement de cette dernière aux télomères et exerce, de ce fait, un effet dominant négatif sur la protection des télomères. La forme tronquée d'Apollo perturbe la réplication des télomères : elle induit des aberrations télomériques (doublets et fusions télomériques), créant des intermédiaires anormaux de réplication et perturbant la synthèse du brin retard.

Initialement, Apollo avait été décrit comme un facteur impliqué dans la réparation de certaines lésions de I'ADN (essentiellement des ponts interbrins pouvant être produits par la mitomycine C [MMC]) [34]. Néanmoins, les cellules exprimant la forme tronquée d'Apollo réparent normalement ces lésions, indiquant que la forme tronquée d'Apollo, bien qu'exerçant un effet dominant négatif sur la protection des télomères, ne perturbe pas la réparation des lésions de l'ADN localisées ailleurs dans le génome. Globalement, cette étude a permis d'identifier une nouvelle cause moléculaire de syndrome $\mathrm{HH}$ et de préciser le rôle crucial d'Apollo chez l'homme dans la protection des télomères au cours de la réplication.

Parallèlement à l'étude du facteur Apollo, l'analyse du phénotype cellulaire d'autres patients $\mathrm{HH}$ a révélé l'existence, pour certains d'entre eux, de 


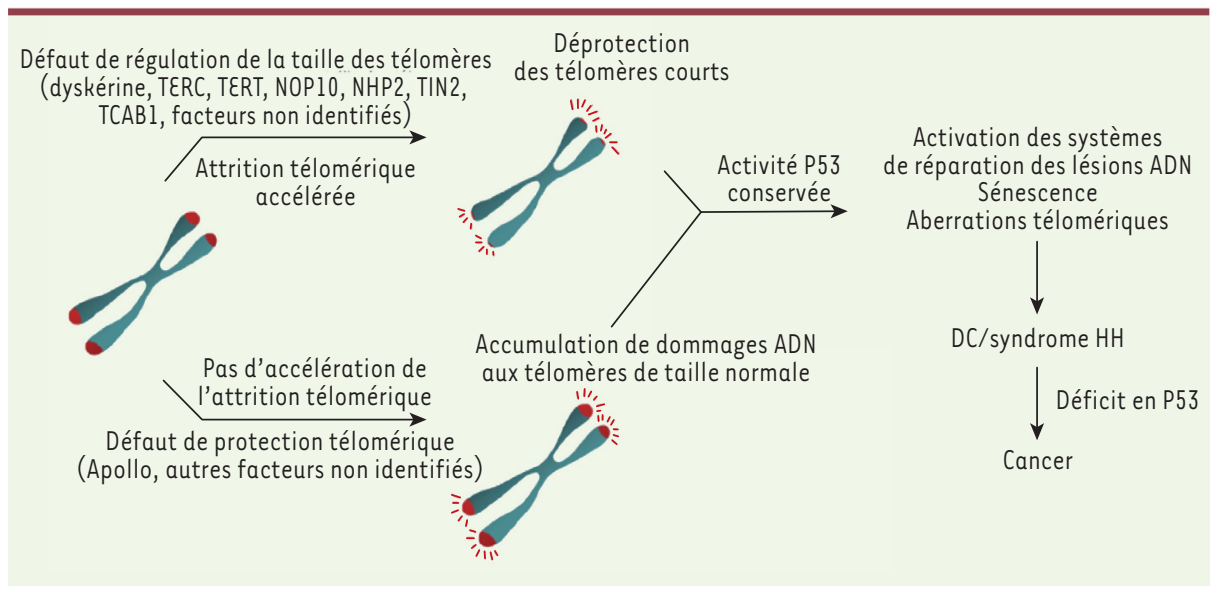

Figure 4. Modèle proposant les différentes origines moléculaires conduisant à l'induction d'un phénotype cellulaire de dyskératose congénitale ou de syndrome de Hoyeraal-Hreidarsson. La présence de TIF et d'une sénescence cellulaire précoce sont des caractéristiques communes des cellules de patients atteints de $\mathrm{DC} / \mathrm{HH}$, et indiquent l'existence d'un dysfonctionnement des télomères. Le dysfonctionnement télomérique peut provenir d'un défaut d'un facteur impliqué dans la régulation de la taille des télomères, aboutissant à

une déprotection télomérique par réduction excessive de la longueur télomérique, comme observée dans les cellules de patients déficients en dyskérine, TERC, TERT, TIN2 ou TCAB1. Le dysfonctionnement télomérique peut aussi résulter d'un défaut d'un facteur de protection télomérique, menant à l'accumulation de dommages à l'ADN aux télomères sans attrition télomérique, comme observée chez le patient exprimant la forme tronquée d’Apollo. Quelle que soit sa cause, le dysfonctionnement télomérique provoqué est responsable du phénotype de DC et de syndrome HH. Le développement d’un cancer chez ces patients peut se produire lorsque la voie p53, responsable de la sénescence et/ou de l'apoptose, est devenue déficiente.

dysfonctionnements télomériques originaux. L'analyse en FISH et COFISH a mis en évidence des aberrations télomériques diverses dont certaines inédites chez l'homme (signaux télomériques interstitiels, perte de signaux télomériques, signaux télomériques multiples) (Figure 3B). Certaines de ces anomalies résultent vraisemblablement d'un défaut réplicatif des télomères dont la cause moléculaire reste à déterminer. Enfin, et de façon inattendue, chez certains de ces patients $\mathrm{HH}$, la taille des télomères mesurée dans les cellules sanguines et les fibroblastes est comparable à celle de télomères de cellules d'individus sains du même âge, indiquant que le dysfonctionnement télomérique observé dans les cellules de ces patients et leurs caractéristiques cliniques ne sont pas inévitablement associés à une réduction anormale de la taille des télomères. Ceci indique que l'origine de la pathologie chez ces patients concerne certainement un défaut des molécules dédiées à la protection et/ou la réplication des télomères plutôt qu'à la régulation de leur taille. Cette observation a également une conséquence importante sur le diagnostic des patients atteints de $\mathrm{DC} / \mathrm{HH}$, puisqu'elle révèle que la mesure de la longueur des télomères des cellules sanguines, couramment utilisée pour le diagnostic de $D C$ et de syndrome $\mathrm{HH}$, bien qu'informative, ne permet pas de diagnostiquer tous les cas de $\mathrm{DC} / \mathrm{HH}$.

L'ensemble de ces données suggèrent que les caractéristiques communes aux DC et aux syndromes HH seraient l'existence d'une sénescence cellulaire précoce induite soit par des télomères anormalement courts, soit par des télomères endommagés mais de taille normale (modèle proposé dans la Figure 4). Une étude génétique systématique des patients atteints de $\mathrm{DC} / \mathrm{HH}$ par les méthodes récemment développées de séquençage à haut débit combinées à des stratégies de filtrage adaptées - comme l'étude de la ségrégation des marqueurs polymorphes dans le cas de familles consanguines - devrait permettre prochainement l'identification des facteurs en cause dans les cas de $\mathrm{DC} / \mathrm{HH}$ encore non élucidés sur le plan moléculaire. Ainsi, ces avancées permettront d'accroître notre connaissance de la physiologie des télomères et pro- bablement d'améliorer la compréhension et la prise en charge de ces maladies. $\diamond$

\section{SUMMARY}

Dyskeratosis congenita: short telomeres are not the rule

Telomeres are nucleoprotein structures at the end of linear chromosomes. Their length, structure, and integrity are regulated by the telomerase complex, the shelterins and components of the DNA damage response. In human subjects, defects in telomere maintenance are responsible for Dyskeratosis Congenita (DC), a rare genetic disorder characterized by aplastic anaemia, premature aging and predisposition to cancer. Recent data from the study of patients with HoyeraalHreidarsson syndrome, a severe variant of DC, demonstrate the great molecular heterogeneity of this disease. While most cases of DC are associated with defects in factors involved in telomere length regulation, some severe forms of the disease seem to be rather associated with defects in telomere replication and protection. $\diamond$

\section{LIENS D’INTÉRÊT}

Les auteurs déclarent n'avoir aucun lien d'intérêt concernant les données publiées dans cet article.

\section{RéFÉRENCES}

1. O'Sullivan RJ, Karlseder J. Telomeres: protecting chromosomes against genome instability. Nat Rev Mol Cell Biol 2010 ; 11 : 171-81.

2. Shay JW, Wright WE. Telomeres and telomerase in normal and cancer stem cells. FEBS Lett $2010 ; 584: 3819-25$.

3. Londoño-Vallejo A, Lenain C, Gilson $\varepsilon$. Cibler les télomères pour forcer les cellules cancéreuses à rentrer en sénescence. Med Sci (Paris) 2008 ; 24 : 383-9. 


\section{RÉFÉRENCES}

4. Bodnar AG, Ouellette M, Frolkis M, et al. Extension of life-span by introduction of telomerase into normal human cells. Science $1998 ; 279$ : 349-52.

5. Shay JW, Bacchetti S. A survey of telomerase activity in human cancer. Eur J Cancer 1997 ; 33 : 787-91.

6. De Lange T. Shelterin: the protein complex that shapes and safeguards human telomeres. Genes Dev $2005 ; 19: 2100-10$.

7. Dokal I. Dyskeratosis congenita in all its forms. Br J Haematol $2000 ; 110: 768-79$.

8. Hoareau-Aveilla C, Henry Y, Leblanc T. La dyskératose congénitale : une maladie méconnue due à un maintien défectueux des télomères. Med Sci (Paris) 2008 ; $24: 390-8$.

9. Hreidarsson $\mathrm{S}$, Kristjansson $\mathrm{K}$, Johannesson $\mathrm{G}$, et al. A syndrome of progressive pancytopenia with microcephaly, cerebellar hypoplasia and growth failure. Acta Paediatr Scand $1988 ; 77$ : 773-5.

10. Mitchell JR, Wood $\varepsilon$, Collins K. A telomerase component is defective in the human disease dyskeratosis congenita. Nature $1999 ; 402: 551-5$.

11. Heiss NS, Knight SW, Vulliamy TJ, et al. X-linked dyskeratosis congenita is caused by mutations in a highly conserved gene with putative nucleolar functions. Nat Genet $1998 ; 19: 32-8$.

12. Rashid R, Liang B, Baker DL, et al. Crystal structure of a Cbf5-Nop10-Garl complex and implications in RNA-guided pseudouridylation and dyskeratosis congenita. Mol Cell $2006 ; 21: 249-60$.

13. Zeng XL, Thumati NR, Fleisig HB, et al. The accumulation and not the specific activity of telomerase ribonucleoprotein determines telomere maintenance deficiency in $\mathrm{X}$-linked dyskeratosis congenita. Hum Mol Genet 2011 ; 21 : 721-9.

14. Vulliamy T, Marrone A, Goldman F, et al. The RNA component of telomerase is mutated in autosomal dominant dyskeratosis congenita. Nature $2001 ; 413: 432-5$.

15. Ly H, Calado RT, Allard P, et al. Functional characterization of telomerase RNA variants found in patients with hematologic disorders. Blood $2005 ; 105$ : 2332-9.

16. Du HY, Idol R, Robledo $S$, et al. Telomerase reverse transcriptase haploinsufficiency and telomere length in individuals with 5p-syndrome. Aging Cell $2007 ; 6: 689-97$.

17. Savage SA, Giri N, Baerlocher GM, et al. TINF2, a component of the shelterin telomere protection complex, is mutated in dyskeratosis congenita. Am J Hum Genet $2008 ; 82: 501-9$.

18. Canudas $S$, Houghtaling BR, Bhanot $M$, et al. A role for heterochromatin protein $l \gamma$ at human telomeres. Genes Dev $2011 ; 25: 1807-19$.

19. Yang $\mathrm{D}$, He $\mathrm{O}$, Kim H, et al.TIN2 protein dyskeratosis congenita missense mutants are defective in association with telomerase. J Biol Chem 2011 ; 286 : 23022-30.

20. Walne AJ, Vulliamy T, Marrone A, et al. Genetic heterogeneity in autosomal recessive dyskeratosis congenita with one subtype due to mutations in the telomerase-associated protein NOP10. Hum Mol Genet 2007 ; $16: 1619-29$.

21. Vulliamy T, Beswick R, Kirwan M, et al. Mutations in the telomerase component NHP2 cause the premature ageing syndrome dyskeratosis congenita. Proc Natl Acad Sci USA 2008 ; 105 : 8073-8.

22. Zhong F, Savage SA, Shkreli M, et al. Disruption of telomerase trafficking by TCABl mutation causes dyskeratosis congenita. Genes Dev $2011 ; 25: 11-6$.

23. Walne AJ, Vulliamy T, Beswick R, et al. Mutations in $\mathrm{Cl} 16$ orf57 and normal-length telomeres unify a subset of patients with dyskeratosis congenita, poikiloderma with neutropenia and RothmundThomson syndrome. Hum Mol Genet 2010 ; 19 : 4453-61.
24. Du HY, Pumbo E, Ivanovich J, et al. TERC and TERT gene mutations in patients with bone marrow failure and the significance of telomere length measurements. Blood $2009 ; 113: 309-16$.

25. Aviv A, Hunt SC, Lin J, et al. Impartial comparative analysis of measurement of leukocyte telomere length/DNA content by Southern blots and qPCR. Nucleic Acids Res 2011 ; 39 : el34.

26. Alter BP, Baerlocher GM, Savage SA, et al. Very short telomere length by flow fluorescence in situ hybridization identifies patients with dyskeratosis congenita. Blood 2007 ; 110 : 1439-47.

27. Vulliamy T, Marrone A, Szydlo R, et al. Disease anticipation is associated with progressive telomere shortening in families with dyskeratosis congenita due to mutations in TERC. Nat Genet 2004 ; $36: 447-9$.

28. Vulliamy TJ, Kirwan MJ, Beswick R, et al. Differences in disease severity but similar telomere lengths in genetic subgroups of patients with telomerase and shelterin mutations. PLoS One 2011; 6 : e24383.

29. Alter BP, Rosenberg PS, Giri N, et al. Telomere length is associated with disease severity and declines with age in dyskeratosis congenita. Haematologica $2011 ; 97: 353-9$.

30. Touzot F, Gaillard L, Vasquez N, et al. Heterogeneous telomere defects in patients with severe forms of dyskeratosis congenita. J Allergy Clin Immunol 2011 ; 129 : 473-82.

31. Revy P, Busslinger M, Tashiro K, et al. A syndrome involving intrauterine growth retardation, microcephaly, cerebellar hypoplasia, B lymphocyte deficiency, and progressive pancytopenia. Pediatrics 2000 ; 105 : ع39.

32. Touzot F, Callebaut I, Soulier J, et al. Function of Apollo (SNMIB) at telomere highlighted by a splice variant identified in a patient with HoyeraalHreidarsson syndrome. Proc Natl Acad Sci USA 2010 ; 107 : 10097-102.

33. Ye J, Lenain C, Bauwens S, et al. TRF2 and apollo cooperate with topoisomerase 2 alpha to protect human telomeres from replicative damage. Cell $2010 ; 142: 230-42$.

34. Demuth I, Digweed M, Concannon P. Human SNMIB is required for normal cellular response to both DNA interstrand crosslink-inducing agents and ionizing radiation. Oncogene $2004 ; 23: 8611-8$.

35. Zinsser F. Atrophia cutis reticularis cum pigtnentatione, dystrophia unguim et leukuplakia oris. Ikonographia Dermatol (Hyoto) $1910 ; 5: 219-23$.

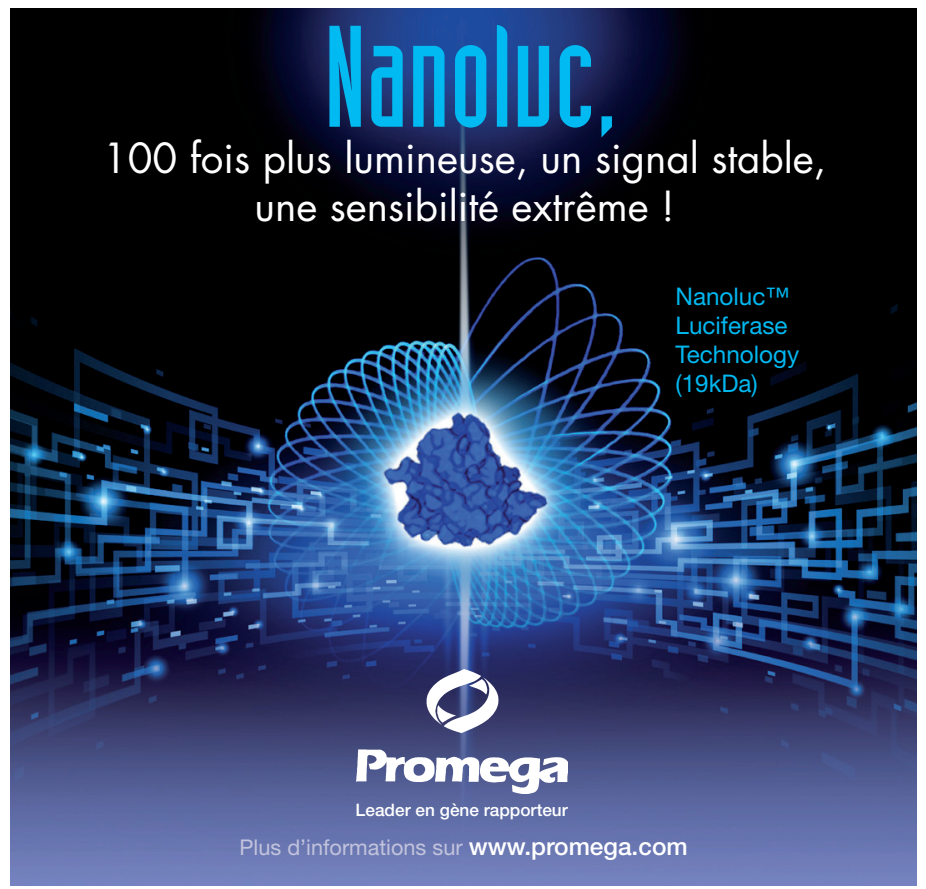

TIRÉS À PART

P. Revy 